\title{
Estimation of Seasonal Cointegration under Conditional Heteroskedasticity
}

\author{
Byeongchan Seong ${ }^{1, a}$ \\ ${ }^{a}$ Department of Applied Statistics, Chung-Ang University, Korea
}

\begin{abstract}
We consider the estimation of seasonal cointegration in the presence of conditional heteroskedasticity $(\mathrm{CH})$ using a feasible generalized least squares method. We capture cointegrating relationships and time-varying volatility for long-run and short-run dynamics in the same model. This procedure can be easily implemented using common methods such as ordinary least squares and generalized least squares. The maximum likelihood (ML) estimation method is computationally difficult and may not be feasible for larger models. The simulation results indicate that the proposed method is superior to the ML method when $\mathrm{CH}$ exists. In order to illustrate the proposed method, an empirical example is presented to model a seasonally cointegrated times series under $\mathrm{CH}$.

Keywords: seasonal error correction model, seasonal unit root, reduced rank estimation, multivariate $\mathrm{GARCH}$, feasible generalized least squares, maximum likelihood estimation, vector autoregressive model
\end{abstract}

\section{Introduction}

The seminal work of Engle and Granger (1987) allowed statistical methods to be applied to the analysis of cointegrated systems. Among others, the joint modeling of cointegration and generalized autoregressive conditional heteroskedasticity (GARCH), proposed by Bollerslev (1986) has attracted the attention of many researchers in finance and economics because the same model can explain two common economic variable characteristics of long-run equilibrium and time-varying volatility. For example, Seo (2007) developed the asymptotic distribution of the cointegrating vector estimator in the vector error correction model (VECM) with GARCH errors. Herwartz and Lütkepohl (2011) proposed a feasible generalized least squares (FGLS) estimator in the joint model and showed that it has superior small sample properties. However, all these studies are restricted to nonseasonal cointegration.

The VECM for seasonal cointegration is more complicated and has more parameters than nonseasonal cointegration. This is because the former has more than two error correction terms, composed of several seasonal filters including complex unit roots; see, for example, Seong (2013) and the references cited therein. Therefore, if we use a full maximum likelihood (ML) procedure that takes into account the GARCH errors, computation of the estimates will be quite demanding, and it may even be infeasible for larger models with a moderate number of variables and a realistic number of lags.

We explore the estimation of a seasonal VECM with conditional heteroskedasticity $(\mathrm{CH})$ using a FGLS method that is easier to implement than the ML method. The proposed method relies on common linear regression methods, such as ordinary least squares (OLS) and generalized least squares

\footnotetext{
${ }^{1}$ Corresponding author: Department of Applied Statistics, Chung-Ang University, 221, Heukseok-dong, Dongjak-gu, Seoul 06974, Korea. E-mail: bcseong@ @au.ac.kr

Published 30 November 2015 / journal homepage: http://csam.or.kr

(c) 2015 The Korean Statistical Society, and Korean International Statistical Society. All rights reserved.
} 
(GLS), which do not require numerical optimization; subsequently, all the computations are based on closed form expressions. Monte Carlo experiments are conducted to evaluate the proposed procedure and for an empirical illustration, we analyze monthly U.S. Housing Starts and Sold using the procedure.

\section{The Model}

We consider a vector autoregressive (VAR) model for a $K$-dimensional process $Y_{t}$ satisfying

$$
\Pi(L) Y_{t}=\left(I_{K}-\sum_{j=1}^{p} \Pi_{j} L^{j}\right) Y_{t}=\varepsilon_{t}, \quad \text { for } t=1, \ldots, T,
$$

where $I_{K}$ denotes a $K \times K$ identity matrix, $\Pi(L)$ is a polynomial matrix, and $L$ is a lag operator such that $L^{j} Y_{t}=Y_{t-j}$. To allow for the possible presence of $\mathrm{CH}$, the error process $\varepsilon_{t}$ is assumed to be a vector martingale difference sequence with $E\left(\varepsilon_{t} \mid \mathcal{F}_{t-1}\right)=0$ and $E\left(\varepsilon_{t} \varepsilon_{t}^{\prime} \mid \mathcal{F}_{t-1}\right)=\Omega_{t}$, where $\mathcal{F}_{t}$ is the $\sigma$-field generated by $Y_{t}, Y_{t-1}, \ldots$. We assume that the roots of the determinant $|\Pi(z)|=0$ are on or outside the unit circle and that $Y_{t}$ is observed on a quarterly basis and has no deterministic terms. Models with other seasonal periods (e.g., monthly) and models with deterministic terms that may contain a constant, a linear trend, or seasonal dummies can easily be implemented as in Ahn et al. (2004).

Then, as in Ahn et al. (2004), if the series are cointegrated of order $(1,1)$ at frequencies $0, \pi, \pi / 2$, and $3 \pi / 2$, model $(2.1)$ can be rewritten as the following seasonal VECM:

$$
\Psi(L) Z_{t}=A_{1} B_{1} U_{t-1}+A_{2} B_{2} V_{t-1}+\left(A_{3} B_{4}+A_{4} B_{3}\right) W_{t-1}+\left(A_{4} B_{4}-A_{3} B_{3}\right) W_{t-2}+\varepsilon_{t},
$$

where $Z_{t}=\left(1-L^{4}\right) Y_{t}, U_{t}=(1+L)\left(1+L^{2}\right) Y_{t}, V_{t}=(1-L)\left(1+L^{2}\right) Y_{t}, W_{t}=\left(1-L^{2}\right) Y_{t}, \Psi(L)$ is a matrix polynomial of order $p-4, A_{j}$ and $B_{j}$ are $K \times r_{j}$ and $r_{j} \times K$ matrices, respectively, with rank equal to $r_{j}$ for $j=1, \ldots, 4$, and $r_{3}=r_{4}$. For a unique parameterization, we need to normalize the $B_{j}$ s such that $B_{1}=\left[I_{r_{1}}, B_{10}\right], B_{2}=\left[I_{r_{2}}, B_{20}\right], B_{3}=\left[I_{r_{3}}, B_{30}\right]$, and $B_{4}=\left[O_{r_{3}}, B_{40}\right]$, where $O_{r_{j}}$ is an $r_{j} \times r_{j}$ matrix of zeros, and the $B_{j 0} \mathrm{~s}$ are $r_{j} \times\left(K-r_{j}\right)$ matrices of unknown parameters. Lütkepohl (2005) explained that this normalization does not imply a loss of generality from a practical point of view. Note that $r_{1}, r_{2}$, and $r_{3}\left(r_{4}\right)$ denote the seasonal cointegrating ranks at frequencies $0, \pi$, and $\pi / 2(3 \pi / 2)$, respectively, and that $B_{1} U_{t}, B_{2} V_{t},\left(B_{3}+B_{4} L\right) W_{t}$, and $\left(B_{4}-B_{3} L\right) W_{t}$ are stationary processes, that is, they represent cointegrating relationships or long-run equilibrium relationships.

\section{Estimation of a Seasonal VECM with $\mathrm{CH}$}

Before we present a FGLS method for estimating seasonal cointegration under $\mathrm{CH}$, we consider a Gaussian ML estimation.

\subsection{ML estimation}

For a sample with $T$ observations and $p$ presample values, ML estimation of VECM (2.2) is theoretically straightforward if we assume $\varepsilon_{t} \mid \mathcal{F}_{t-1} \sim N\left(0, \Omega_{t}\right)$. Collecting all parameters in a vector $\eta$, the log-likelihood function is given by

$$
l(\eta)=T^{-1} \sum_{t=1}^{T} l_{t}(\eta), \quad \text { where } l_{t}(\eta)=-\frac{1}{2} \log \left|\Omega_{t}(\eta)\right|-\frac{1}{2} \varepsilon_{t}(\eta)^{\prime} \Omega_{t}(\eta)^{-1} \varepsilon_{t}(\eta)
$$


Therefore, full ML estimator with allowance for $\mathrm{CH}$ (FML) can be obtained by maximizing the function. Ahn and Reinsel (1994) obtained the ML estimator ignoring $\mathrm{CH}$, that is, assuming $\varepsilon_{t} \sim N(0, \Omega)$, by the Gaussian reduced rank ML estimation (RRML).

\subsection{A FGLS estimation}

Given the practical difficulties in computing the ML estimator in the presence of $\mathrm{CH}$, we propose the following three-step procedure for a FGLS estimator.

Step 1. Estimate the parameters in the model

$$
\Psi(L) Z_{t}=C_{1} U_{t-1}+C_{2} V_{t-1}+C_{3} W_{t-1}+C_{4} W_{t-2}+\varepsilon_{t}
$$

by OLS and denote the residuals by $\hat{\varepsilon}_{t}$. Then, estimate the $\mathrm{CH}$ parameters, say $\theta$, from a pseudo ML estimation based on maximizing $\hat{l}(\theta)=T^{-1} \sum_{t=1}^{T} \hat{l}_{t}(\theta)$, where

$$
\hat{l}_{t}(\theta)=-\frac{1}{2} \log \left|\Omega_{t}(\theta)\right|-\frac{1}{2} \hat{\varepsilon}_{t}^{\prime} \Omega_{t}(\theta)^{-1} \hat{\varepsilon}_{t}
$$

Denote the resulting estimate by $\hat{\theta}$ and define $\hat{\Omega}_{t}=\Omega_{t}(\hat{\theta})$.

Step 2. Obtain a feasible estimator $\hat{\alpha}$ for $\alpha=\operatorname{vec}\left(C_{1}, C_{2}, C_{3}, C_{4}, \Psi_{1}, \ldots, \Psi_{p-4}\right)$ from the transformed model, which is derived by premultiplying VECM (2.2) by the $\hat{\Omega}_{t}^{-1 / 2}$ from Step 1 ,

$$
\hat{\Omega}_{t}^{-\frac{1}{2}} Z_{t}=\left[P_{t} \otimes \hat{\Omega}_{t}^{-\frac{1}{2}}\right] \alpha+\tilde{\varepsilon}_{t}
$$

where $P_{t}=\left[U_{t-1}^{\prime}, V_{t-1}^{\prime}, W_{t-1}^{\prime}, W_{t-2}^{\prime}, Z_{t-1}^{\prime}, \ldots, Z_{t-p+4}^{\prime}\right], \tilde{\varepsilon}_{t}=\hat{\Omega}_{t}^{-1 / 2} \varepsilon_{t}$ and $\operatorname{vec}(\cdot)$ vectorizes a matrix columnwise from left to right, and $\otimes$ denotes the Kronecker product. The FGLS estimator $\hat{\alpha}$ is then given by

$$
\hat{\alpha}=\operatorname{vec}\left(\hat{C}_{1}, \hat{C}_{2}, \hat{C}_{3}, \hat{C}_{4}, \hat{\Psi}_{1}, \ldots, \hat{\Psi}_{p-4}\right)=\left[\sum_{t=1}^{T}\left(P_{t}^{\prime} P_{t} \otimes \hat{\Omega}_{t}^{-1}\right)\right]^{-1} \operatorname{vec}\left\{\sum_{t=1}^{T}\left(\hat{\Omega}_{t}^{-1} Z_{t} P_{t}\right)\right\} .
$$

Then, we can obtain $\hat{A}_{1}=\hat{C}_{11}, \hat{A}_{2}=\hat{C}_{12}, \hat{A}_{3}=-\hat{C}_{14}$, and $\hat{A}_{4}=\hat{C}_{13}$ as the FGLS estimators for $A_{1}$, $A_{2}, A_{3}$, and $A_{4}$, respectively, where $\hat{C}_{1 j}$ is the matrix with the first $r_{j}$ columns of $\hat{C}_{j}$ for $j=1,2,3,4$, by using the following relationships between models (2.2) and (3.2).

$$
C_{j}= \begin{cases}A_{j} B_{j}=\left[A_{j}, A_{j} B_{j 0}\right], & \text { for } j=1,2, \\ A_{3} B_{4}+A_{4} B_{3}=\left[A_{4}, A_{3} B_{40}+A_{4} B_{30}\right], & \text { for } j=3, \\ A_{4} B_{4}-A_{3} B_{3}=\left[-A_{3},-A_{3} B_{30}+A_{4} B_{40}\right], & \text { for } j=4 .\end{cases}
$$

If there is no $\mathrm{CH}$ so that $\Omega_{t}=\Omega$, the FGLS estimator reduces precisely to the RRML estimator considered by Ahn and Reinsel (1994). As usual, the covariance matrix of $\hat{\alpha}$ is calculated by $\operatorname{var}(\hat{\alpha})=$ $\left[\sum_{t=1}^{T}\left(P_{t}^{\prime} P_{t} \otimes \hat{\Omega}_{t}^{-1}\right)\right]^{-1}$.

Step 3. In order to compute the FGLS estimator $\hat{\beta}$ for $\beta=\operatorname{vec}\left(B_{10}, B_{20}, B_{30}, B_{40}\right)$, we take a multivariate linear regression model (obtained from the model in (3.3) by replacing the $A_{j} \mathrm{~s}$ and the $\Psi_{j} \mathrm{~s}$ by their respective FGLS estimators, $\hat{A}_{j} \mathrm{~s}$ and $\left.\hat{\Psi}_{j} \mathrm{~s}\right)$. Thus,

$$
\tilde{Z}_{t}=\hat{\Omega}_{t}^{-\frac{1}{2}} Q_{t} \beta+\tilde{\varepsilon}_{t}
$$


where

$$
\begin{aligned}
& \tilde{Z}_{t}=\hat{\Omega}_{t}^{-\frac{1}{2}}\left(Z_{t}-\hat{A}_{1} U_{1 t-1}-\hat{A}_{2} V_{1 t-1}-\hat{A}_{4} W_{1 t-1}+\hat{A}_{3} W_{1 t-2}-\sum_{j=1}^{p-4} \hat{\Psi}_{j} Z_{t-j}\right), \\
& Q_{t}=\left[U_{2 t-1}^{\prime} \otimes \hat{A}_{1}, V_{2 t-1}^{\prime} \otimes \hat{A}_{2}, W_{2 t-1}^{\prime} \otimes \hat{A}_{4}-W_{2 t-2}^{\prime} \otimes \hat{A}_{3}, W_{2 t-1}^{\prime} \otimes \hat{A}_{3}+W_{2 t-2}^{\prime} \otimes \hat{A}_{4}\right],
\end{aligned}
$$

$U_{1 t}, V_{1 t}$, and $W_{1 t}$ are the first $r_{1}, r_{2}$, and $r_{3}$ components of $U_{t}, V_{t}$, and $W_{t}$, respectively, and $U_{2 t}, V_{2 t}$, and $W_{2 t}$ are the last $K-r_{1}, K-r_{2}$, and $K-r_{3}$ components of $U_{t}, V_{t}$, and $W_{t}$, respectively. Then, the FGLS estimator for $\beta$ can be expressed by

$$
\hat{\beta}=\left(\sum_{t=1}^{T} Q^{\prime}{ }_{t} \hat{\Omega}_{t}^{-1} Q_{t}\right)^{-1}\left(\sum_{t=1}^{T} Q^{\prime}{ }_{t} \hat{\Omega}_{t}^{-1} \dot{Z}_{t}\right),
$$

where $\dot{Z}_{t}=Z_{t}-\hat{A}_{1} U_{1 t-1}-\hat{A}_{2} V_{1 t-1}-\hat{A}_{4} W_{1 t-1}+\hat{A}_{3} W_{1 t-2}-\sum_{j=1}^{p-4} \hat{\Psi}_{j} Z_{t-j}$. Similarly in Step 2, if there is no $\mathrm{CH}$, the FGLS estimator reduces to the estimator in Ahn and Reinsel (1994). The covariance matrix of $\hat{\beta}$ is calculated as $\operatorname{var}(\hat{\beta})=\left(\sum_{t=1}^{T} Q^{\prime}{ }_{t} \hat{\Omega}_{t}^{-1} Q_{t}\right)^{-1}$.

\section{Monte Carlo Simulations}

Monte Carlo simulations are conducted to evaluate the finite sample properties of the FGLS estimators suggested in this article. We compare these properties with those of the two ML-based estimators, FML and RRML, which are explained in Section 3.1.

We consider a bivariate data-generating process (DGP) as follows:

$$
Z_{t}=-A_{3} B_{3} W_{t-2}+\varepsilon_{t},
$$

where $Z_{t}=\left(1-L^{4}\right) Y_{t}, A_{3}=\left(0, a_{3}\right)^{\prime}=(0,0.5)^{\prime}$ and $B_{3}=\left(1, b_{3}\right)=(1,-1)$. We also assume $\varepsilon_{t}=L e_{t}$ and $E\left(e_{t} e_{t}^{\prime} \mid \mathcal{F}_{t-1}\right)=\Sigma_{t}$, where

$$
\begin{gathered}
L=\left(\begin{array}{cc}
1 & 0 \\
\lambda & 1
\end{array}\right), \quad \Sigma_{t}=\left(\begin{array}{cc}
\sigma_{1 t}^{2} & 0 \\
0 & \sigma_{2 t}^{2}
\end{array}\right), \\
\sigma_{j t}^{2}=\omega_{j}+\psi_{j} e_{j, t-1}^{2}+\phi_{j} \sigma_{j, t-1}^{2}, \quad e_{j t}=\sigma_{j t} \xi_{j t}, \quad \text { and } \quad \xi_{j t} \sim N(0,1) \quad \text { for } j=1,2 .
\end{gathered}
$$

The $Y_{t}$ s in equation (4.1) are seasonally cointegrated with a cointegrating rank of one only at frequency $\pi / 2, \varepsilon_{t}$ is a generalized orthogonal (GO-) GARCH process (van der Weide, 2002), and $\lambda$ determines the correlation between the two components of $\varepsilon_{t}$. Note that $Y_{t}$ is cointegrated at a single seasonal frequency but if one performs a usual cointegration analysis by Johansen (1988), we cannot obtain the correct cointegrating relationship. It is related to a different form of VECM for seasonal cointegration.

The cointegrating parameter $b_{3}=-1$ is in line with typical parameter in economic models. Table 1 summarizes the of values of the parameters $\lambda, \psi_{j}$ and $\phi_{j}$ used in the simulations. As in most of the DGPs in Seo (2007) and Herwartz and Lütkepohl (2011), we choose identical values for the GARCH parameters of the two error components, and we set the value of $\psi_{j}+\phi_{j}$ to be close to unity. It is suitable for investigating possible estimation efficiency losses due to model misspecification since the case of $\left(\psi_{j}, \phi_{j}\right)=(0,0)$ has no GARCH and assumes GARCH when there is none. Note that for higher frequency data, the GARCH parameter $\psi_{j}$ tends to be closer to zero while $\phi_{j}$ is often close to unity. 
Table 1: Parameter values used in Monte Carlo simulations

\begin{tabular}{crcc}
\hline \hline DGPs & $\lambda$ & $\psi_{j}$ & $\phi_{j}$ \\
\hline 1 & 0 & 0 & 0 \\
2 & -0.5 & 0.10 & 0.85 \\
3 & 0.5 & 0.10 & 0.85 \\
4 & -0.5 & 0.25 & 0.70 \\
5 & 0.5 & 0.25 & 0.70 \\
6 & -0.5 & 0.40 & 0.55 \\
7 & 0.5 & 0.40 & 0.55 \\
8 & & BEKK & \\
9 & & DCC & \\
\hline \hline
\end{tabular}

DGP = data-generating process.

For GARCH processes which do not have a GO-GARCH structure, we consider two more multivariate GARCH processes. DGP 8 is a BEKK model (Engle and Kroner, 1995), i.e.,

$$
\Omega_{t}=D D^{\prime}+F \varepsilon_{t} \varepsilon_{t}^{\prime} F^{\prime}+H \Omega_{t-1} H^{\prime}
$$

with

$$
D=\left(\begin{array}{cc}
2.5 \times 10^{-3} & 0 \\
-8.4 \times 10^{-4} & 8.3 \times 10^{-5}
\end{array}\right), \quad F=\left(\begin{array}{rr}
0.229 & -0.173 \\
0.005 & 0.174
\end{array}\right), \quad \text { and } \quad H=\left(\begin{array}{rr}
0.954 & 0.033 \\
0.008 & 0.981
\end{array}\right) \text {. }
$$

The parameter values are identical to those used in Herwartz and Lütkepohl (2011). Note that this BEKK process has high persistence in second moments but remains covariance stationary.

As another GARCH process, DGP 9 is a dynamic conditional correlation (DCC) model where the volatility matrix of $\varepsilon_{t}, \Omega_{t}$, is defined by

$$
\Omega_{t}=D_{t} \rho_{t} D_{t},
$$

where $D_{t}=\operatorname{diag}\left(\sigma_{1 t}, \sigma_{2 t}\right)$ and $\sigma_{j t}(j=1,2)$ follows $\operatorname{GARCH}(1,1)$ with $\left(\psi_{1}, \phi_{1}\right)=\left(\psi_{2}, \phi_{2}\right)=(0.1,0.85)$ as in equation (4.3). The conditional correlation matrix $\rho_{t}$ is defined as

$$
\begin{gathered}
\rho_{t}=\operatorname{diag}\left(J_{t}\right)^{-\frac{1}{2}} J_{t} \operatorname{diag}\left(J_{t}\right)^{-\frac{1}{2}} \\
J_{t}=\left(1-\theta_{1}-\theta_{2}\right) \bar{J}+\theta_{1} s_{t-1} s_{t-1}^{T}+\theta_{2} J_{t-1}, \quad \theta_{1}=0.05, \quad \theta_{2}=0.93,
\end{gathered}
$$

where $\bar{J}$ is the unconditional correlation matrix of $s_{t}=D_{t}^{-1} \varepsilon_{t}$ with $\operatorname{corr}\left(s_{1 t}, s_{2 t}\right)=0.5$. Engle (2002) proposed a DCC model that would divide multivariate volatility processes into volatility series $\sigma_{j t} \mathrm{~s}$ and the correlation matrix $\rho_{t}$.

The series lengths considered are $T=100$ and 200, and the replications are set at 1,000 each. For each DGP, the adjustment parameter $a_{3}$ and the cointegration parameter $b_{3}$ are estimated using the three methods-FGLS, FML, and RRML. To obtain FML, the log-likelihood in (3.1) is maximized using the Berndt-Hall-Hall-Hausman (BHHH) algorithm, which is justified under the assumption of conditional normality (Berndt et al., 1974). Table 2 summarizes the estimators that are computed by the mean squared errors (MSEs) and the mean absolute errors (MAEs).

The MSEs and MAEs in Table 2 show that estimation precision increases with the sample size, reflecting consistency of the estimators. It does not make much difference for the ranking of the estimators whether MSEs or MAEs are considered. A comparison of FGLS (and FML) with RRML shows that it pays to account for GARCH errors. In almost all DGPs where CH is allowed for, FGLS 
Table 2: Simulation results for estimators of $a_{3}$ and $b_{3}$ based on DGPs 1-9: MSE $\left(\times 10^{-3}\right)$ and MAE $\left(\times 10^{-2}\right)$ for three different estimation methods, FGLS, FML and RRML, based on 1,000 replications for each sample size $T$

\begin{tabular}{|c|c|c|c|c|c|c|c|c|c|}
\hline \multirow{3}{*}{ DGP } & \multirow{3}{*}{ Estimators } & \multicolumn{4}{|c|}{$a_{3}$} & \multicolumn{4}{|c|}{$b_{3}$} \\
\hline & & MSE & MAE & MSE & MAE & MSE & MAE & MSE & MAE \\
\hline & & \multicolumn{2}{|c|}{$T=100$} & \multicolumn{2}{|c|}{$T=200$} & \multicolumn{2}{|c|}{$T=100$} & \multicolumn{2}{|c|}{$T=200$} \\
\hline \multirow{3}{*}{1} & FGLS & 4.538 & 5.313 & 2.161 & 3.668 & 2.454 & 3.489 & 0.621 & 1.784 \\
\hline & FML & 4.334 & 5.243 & $\underline{2.098}$ & 3.615 & 2.059 & 3.252 & 0.552 & 1.723 \\
\hline & RRML & 4.323 & 5.235 & $\overline{2.110}$ & $\overline{3.633}$ & 1.972 & 3.197 & $\overline{0.556}$ & $\overline{1.726}$ \\
\hline \multirow{3}{*}{2} & FGLS & $\overline{3.947}$ & $\overline{4.919}$ & $\underline{1.852}$ & $\underline{3.403}$ & $\overline{2.563}$ & $\overline{\mathbf{3 . 5 0 7}}$ & 0.643 & 1.844 \\
\hline & FML & 3.971 & 4.915 & $\overline{1.987}$ & $\overline{3.503}$ & 2.686 & $\overline{3.577}$ & $\overline{0.679}$ & $\overline{1.863}$ \\
\hline & RRML & 3.895 & 4.912 & 1.995 & 3.514 & 2.539 & 3.523 & 0.703 & 1.887 \\
\hline \multirow{3}{*}{3} & FGLS & $\overline{\mathbf{8 . 8 9 9}}$ & $\overline{7.564}$ & 4.829 & 5.467 & $\overline{2.886}$ & 3.589 & 0.529 & 1.734 \\
\hline & FML & $\overline{9.042}$ & $\underline{7.506}$ & $\overline{5.071}$ & $\overline{5.580}$ & 2.832 & $\overline{3.590}$ & $\overline{0.564}$ & $\overline{1.784}$ \\
\hline & RRML & 9.076 & $\overline{7.535}$ & 5.066 & 5.575 & 2.703 & 3.611 & 0.573 & 1.793 \\
\hline \multirow{3}{*}{4} & FGLS & 4.124 & 4.923 & 1.472 & 3.058 & $\overline{3.166}$ & 3.407 & 0.420 & 1.509 \\
\hline & FML & $\overline{4.924}$ & $\overline{5.375}$ & $\overline{2.137}$ & $\overline{3.683}$ & $\overline{3.345}$ & $\overline{3.787}$ & $\overline{0.697}$ & $\overline{1.907}$ \\
\hline & RRML & 5.115 & 5.468 & 2.350 & 3.828 & 3.173 & 3.704 & 0.692 & 1.869 \\
\hline \multirow{3}{*}{5} & FGLS & 9.724 & 7.692 & 4.327 & 5.174 & 2.447 & 3.348 & 0.595 & 1.584 \\
\hline & FML & $\overline{11.945}$ & $\overline{8.437}$ & $\overline{6.654}$ & $\overline{6.337}$ & $\overline{3.032}$ & $\overline{3.735}$ & $\overline{0.963}$ & $\overline{1.973}$ \\
\hline & RRML & 12.146 & 8.443 & 6.915 & 6.409 & 3.533 & 3.781 & 0.801 & 1.919 \\
\hline \multirow{3}{*}{6} & FGLS & $\mathbf{3 . 5 2 2}$ & 4.622 & 1.610 & 3.157 & 2.857 & 3.283 & 0.612 & 1.526 \\
\hline & FML & $\overline{5.853}$ & $\overline{5.734}$ & $\overline{2.788}$ & $\overline{3.974}$ & $\overline{4.420}$ & $\overline{4.205}$ & $\overline{1.819}$ & $\overline{2.671}$ \\
\hline & RRML & 6.511 & 5.871 & 3.507 & 4.359 & 4.045 & 3.905 & 0.872 & 1.966 \\
\hline \multirow{3}{*}{7} & FGLS & $\underline{10.159}$ & 7.917 & 4.745 & $\underline{5.396}$ & 2.045 & 2.940 & 0.528 & 1.541 \\
\hline & FML & $\overline{13.561}$ & $\overline{9.146}$ & $\overline{7.669}$ & $\overline{6.777}$ & $\overline{3.035}$ & $\overline{3.658}$ & $\overline{1.812}$ & $\overline{2.801}$ \\
\hline & RRML & 13.961 & 9.253 & 8.400 & 7.095 & 2.901 & 3.394 & 2.516 & 2.153 \\
\hline \multirow{3}{*}{8} & FGLS & 9.061 & 7.438 & 1.901 & 3.006 & 3.676 & 4.824 & 0.527 & 1.681 \\
\hline & FML & 8.845 & 7.405 & 1.881 & 3.021 & 3.730 & $\overline{4.836}$ & $\overline{0.548}$ & $\overline{1.711}$ \\
\hline & RRML & 8.709 & 7.360 & $\overline{1.903}$ & 3.041 & 3.733 & 4.832 & 0.571 & 1.724 \\
\hline \multirow{3}{*}{9} & FGLS & 9.004 & $\overline{7.380}$ & 1.423 & 2.696 & 3.885 & 4.883 & 0.365 & 1.395 \\
\hline & FML & 8.818 & 7.339 & 1.397 & 2.671 & 3.843 & 4.817 & $\overline{0.372}$ & $\overline{1.421}$ \\
\hline & RRML & $\overline{8.959}$ & $\overline{7.360}$ & $\overline{1.411}$ & 2.685 & 3.823 & 4.786 & 0.369 & 1.409 \\
\hline
\end{tabular}

Note: The underlined bold numbers denote the minimum MSEs or MAEs in estimating $a_{3}$ and $b_{3}$.

DGP = data-generating process; $\mathrm{MSE}=$ mean squared error; $\mathrm{MAE}=$ mean absolute error; FGLS = feasible generalized least squares; FML = full maximum likelihood estimator; RRML = reduced rank maximum likelihood estimation.

is substantially better than both FML and RRML in terms of MSE and MAE. However, FML seems to perform at the same level as RRML, even though the former is theoretically better than the latter.

In DGP 1 where there is no CH, FGLS and FML are expected to perform worse than RRML because of the loss in estimation efficiency associated with these methods. However, poorer performance is observed only for the case with $T=100$. It is remarkable that FML tends to be superior than RRML to estimate the parameters, even when there is no $\mathrm{CH}$. It may imply that the estimation of the VECM parameters are not sensitive to the misspecification of GARCH errors.

Focusing on DGPs 4-7 with GO-GARCH processes, FGLS produces the best performance to estimate the parameters $a_{3}$ and $b_{3}$, irrespective of the sample size. In some cases of DGPs 2 and 3, FGLS performs worse than FML or RRML when $T=100$. We conjecture that, in smaller sample size, the closer the parameter $\phi_{j}$ is to zero, the higher the efficiency of FGLS relative to FML and RRML. This phenomenon may occur because the DGPs have a low (quarterly) frequency. When $T=$ 200, FGLS still shows the best performance. In all cases with GO-GARCH, the positive correlation between the components $(\lambda>0)$ deteriorates the performance of the estimation methods but the sign of $\lambda$ has no effect on the ranking of the estimators.

FGLS performs better than ML in estimating the nonstationary parameter DGPs 8 and 9 when the true GARCH process is not a GO-GARCH type, especially when $T=200$. However, FGLS ap- 


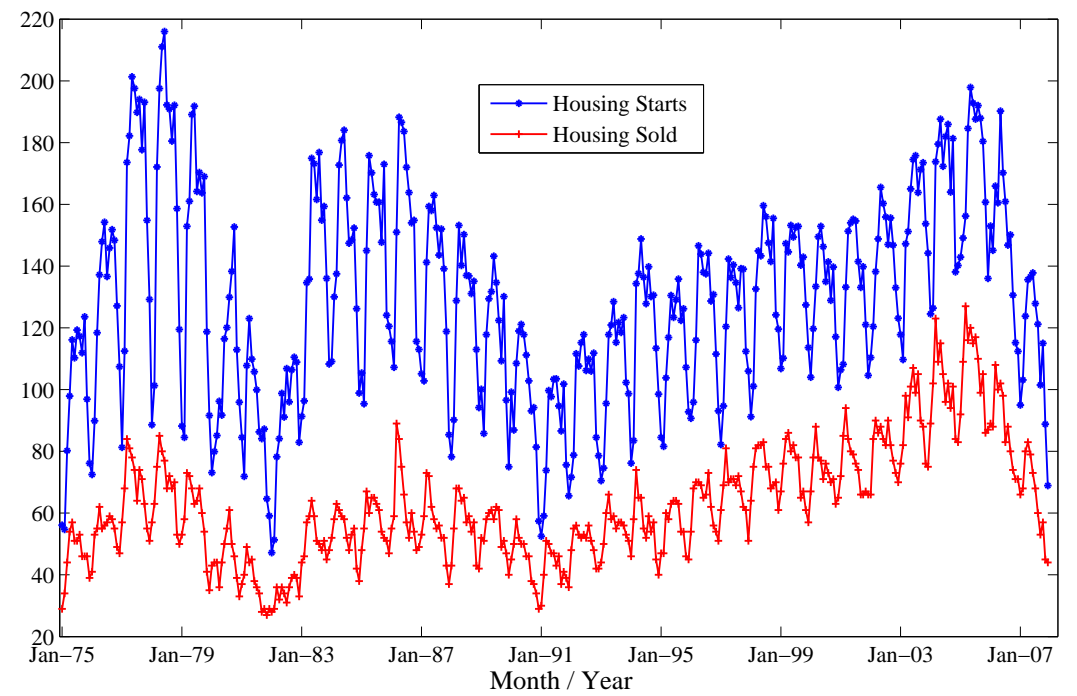

Figure 1: Monthly U.S. Housing Starts and Housing Sold (in thousands) for the period January 1975 to December 2007.

pears more sensitive to misspecification of the GARCH structure than ML in estimating the stationary parameter $a_{3}$ with smaller sample sizes.

In summary, these observations underpin that the FGLS accounting for $\mathrm{CH}$ is overall superior to ML in terms of MSE and MAE. ML with allowance for $\mathrm{CH}$ may produce very unreliable estimates especially if the GARCH assumption is more complex than the true dynamics of the DGP.

\section{An Empirical Example}

In this section, an example is provided to model a seasonally cointegrated time series under $\mathrm{CH}$. We analyze the monthly U.S. Housing Starts $y_{1 t}$ and Housing Sold $y_{2 t}$ (in thousands) for the period January 1975 to December 2007 with data obtained from the U.S. Census Bureau. Figure 1 displays the time series plot of the raw data. We take the natural logarithm of the raw data and identify a model as a VAR(14) with a constant term as:

$$
\begin{aligned}
\left(1-L^{12}\right) Y_{t}= & \sum_{j=1}^{7}\left\{\left(A_{j R} B_{j I}+A_{j I} B_{j R}\right) W_{t-1}^{(j)}+\left(A_{j I} B_{j I}-A_{j R} B_{j R}\right) V_{t-1}^{(j)}\right\} \\
& +\Psi_{1}\left(1-L^{12}\right) Y_{t-1}+\Psi_{2}\left(1-L^{12}\right) Y_{t-2}+\delta+\varepsilon_{t},
\end{aligned}
$$

where $Y_{t}=\left(\log y_{1 t}, \log y_{2 t}\right)^{\prime}$; the subscript $j$ for $j=1, \ldots, 7$ corresponds to seven monthly frequencies $\theta_{j}=(j-1) \pi / 6 ; A_{j R}, A_{j I}, B_{j R}^{\prime}$, and $B_{j I}^{\prime}$ are $2 \times r_{j}$ matrices; $\delta$ is a $2 \times 1$ vector; and $W_{t}^{(j)}$ and $V_{t}^{(j)}$ are the monthly filtered series of $Y_{t}$ with all the seasonal unit roots except $z_{j}=\exp \left(i \theta_{j}\right)$ and $\bar{z}_{j}=\exp \left(-i \theta_{j}\right)$ for $i=\sqrt{-1}$. Note that the $r_{j}$ s denote seasonal cointegrating ranks at frequencies $\theta_{j}=(j-1) \pi / 6$ for $j=1, \ldots, 7$. Ahn et al. (2004) also analyzed similar time series for illustrating the RRML under the homoskedastic Gaussian assumption of $\varepsilon_{t}$.

As an initial step, in order to examine the possible presence of $\mathrm{CH}$, we conduct Engle's ARCH test using the residuals $\hat{\varepsilon}_{t}=\left(\hat{\varepsilon}_{1 t}, \hat{\varepsilon}_{2 t}\right)^{\prime}$ from the estimated model in (5.1) with $r_{j}=2$ for $j=1, \ldots, 7$. The 
Table 3: LR statistics and critical values for identifying seasonal cointegrating ranks in (5.1)

\begin{tabular}{|c|c|c|c|c|c|}
\hline \multirow{2}{*}{$j$} & \multicolumn{2}{|c|}{ LR statistics } & \multicolumn{2}{|c|}{ Critical values at the $5 \%$ level } & \multirow{2}{*}{$\begin{array}{c}\text { Seasonal cointegrating } \\
\text { ranks identified }\end{array}$} \\
\hline & $r_{j}=0$ & $r_{j} \leq 1$ & $r_{j}=0$ & $r_{j} \leq 1$ & \\
\hline 1 & 14.2 & 0.6 & 12.3 & 4.1 & 1 \\
\hline 2 & 53.1 & 3.7 & 20.5 & 6.2 & 1 \\
\hline 3 & 51.0 & 3.8 & 20.5 & 6.2 & 1 \\
\hline 4 & 63.6 & 24.2 & 20.5 & 6.2 & 2 \\
\hline 5 & 73.3 & 49.8 & 20.5 & 6.2 & 2 \\
\hline 6 & 71.0 & 16.8 & 20.5 & 6.2 & 2 \\
\hline 7 & 54.7 & 22.6 & 12.3 & 4.1 & 2 \\
\hline
\end{tabular}

LR = likelihood ratio.

two obtained $p$-values, $5.4 \times 10^{-6}$ and $7.8 \times 10^{-4}$, imply that there exists a statistically significant $\mathrm{CH}$ in the error process. Seasonal cointegrating ranks are identified by computing the likelihood ratio (LR) statistics (Table 3); see Seong et al. (2006) details. Accordingly, under the 5\% level, cointegrating ranks $\left(r_{j}=1\right)$ are found only at frequencies $0, \pi / 6$, and $\pi / 3$ while full ranks $\left(r_{j}=2\right)$ are found in other frequencies.

Assuming that $\varepsilon_{t}$ follows a GO-GARCH in (4.2) and (4.3), we estimate the $\mathrm{CH}$ parameters through the first step explained in Section 3.2 and obtain

$$
\begin{aligned}
\sigma_{1 t}^{2} & =\underset{(<0.0001)}{0.0001}+\underset{(0.2121)}{0.0457} e_{1, t-1}^{2}+\underset{(<0.0001)}{0.9393} \sigma_{1, t-1}^{2}, \\
\sigma_{2 t}^{2} & =\underset{(<0.0001)}{2 \times 10^{-5}}+\underset{(0.0492)}{0.0123} e_{2, t-1}^{2}+\underset{(0.0191)}{0.9823} \sigma_{2, t-1}^{2},
\end{aligned}
$$

where the numbers in parentheses denote the standard errors of the corresponding estimators. The correlation between the error components is estimated as $\hat{\lambda}=\underset{(0.8933)}{0.49}$. It indicates that shocks to volatility have a persistent effect $\sigma_{1 t}^{2}$ and $\sigma_{2 t}^{2}$ since the sums of the ARCH and GARCH coefficients are close to unity.

Through the second and third steps of Section 3.2, we obtain the FGLS estimators for $\alpha$ and $\beta$, which are based on the ranks $\left(r_{1}, \ldots, r_{7}\right)=(1,1,1,2,2,2,2)$. Table 4 shows them with their standard errors in parentheses. We see that a single cointegration vector at the zero frequency exists as $(1,-0.2781)^{\prime}$. At frequency $\theta_{j}=\pi / 6$, the two long-run equilibrium relationships estimated are $(1,-1.0058-1.0669 L)^{\prime}$ and $(-L,-1.0669+1.0058 L)^{\prime}$, which imply polynomial cointegrations at the annual frequency; in addition, the equilibrium relationships at frequency $\theta_{j}=\pi / 3$ are estimated as $(1,-0.7305-1.3233 L)^{\prime}$ and $(-L,-1.3233+0.7305 L)^{\prime}$. Regarding adjustment parameters $A_{j R}$ and $A_{j I}$, the off-diagonal coefficients at frequencies $\theta_{j}=(j-1) \pi / 6$ for $j=4,5,6,7$ are not significant under the $5 \%$ level, with the exception of one coefficient in $A_{5 I}$.

This brief example shows that the proposed FGLS procedure enables us to simultaneously model long-run equilibrium and time-varying volatility in the seasonal VECM. It is also expected that the FGLS will provide more exact forecasts than ML, because the former produces more reliable estimates than the latter, especially when the series has a time-varying volatility.

\section{Conclusion}

In contrast to the case of nonseasonal cointegration, it is not simple to apply the ML method to seasonal cointegration, especially under $\mathrm{CH}$. In this article, we considered a FGLS procedure for the estimation of a seasonal VECM with $\mathrm{CH}$. This procedure can be easily implemented using common methods such as OLS and GLS. We showed that the proposed procedure performs well compared to 
Table 4: FGLS estimators in $(5.1)$ with $\left(r_{1}, \ldots, r_{7}\right)=(1,1,1,2,2,2,2)$

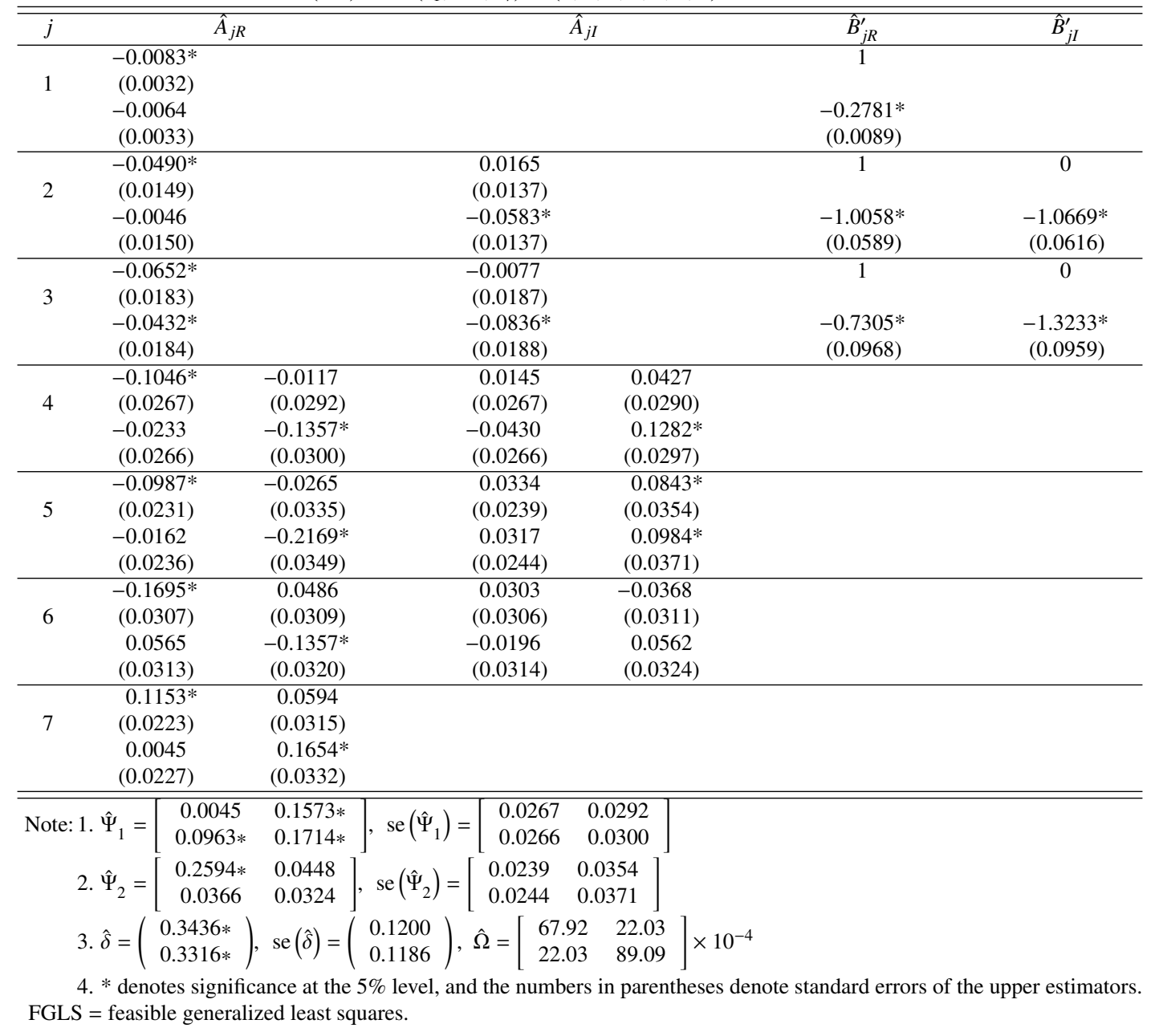

the ML method and we illustrated the procedure with monthly time series data that uses a small-scale Monte Carlo simulation.

\section{References}

Ahn, S. K. and Reinsel, G. C. (1994). Estimation of partially nonstationary vector autoregressive models with seasonal behavior, Journal of Econometrics, 62, 317-350.

Ahn, S. K., Cho, S. and Seong, B. C. (2004). Inference of seasonal cointegration: Gaussian reduced rank estimation and tests for various types of cointegration, Oxford Bulletin of Economics and Statistics, 66, 261-284.

Berndt, E. K., Hall, B. H., Hall, R. E. and Hausman, J. A. (1974). Estimation and inference in nonlinear structural models, Annals of Economic and Social Measurement, 3/4, 653-665.

Bollerslev, T. (1986). Generalized autoregressive conditional heteroskedasticity, Journal of Econometrics, 31, 307-327. 
Engle, R. F. (2002). Dynamic conditional correlation: A simple class of multivariate generalized autoregressive conditional heteroskedasticity models, Journal of Business \& Economic Statistics, 20, 339-350.

Engle, R. F. and Granger, C. W. (1987). Co-integration and error correction: Representation, estimation, and testing, Econometrica, 55, 251-276.

Engle, R. F. and Kroner, K. F. (1995). Multivariate simultaneous generalized ARCH, Econometric Theory, 11, 122-150.

Herwartz, H. and Lütkepohl, H. (2011). Generalized least squares estimation for cointegration parameters under conditional heteroskedasticity, Journal of Time Series Analysis, 32, 281-291.

Johansen, S. (1988). Statistical analysis of cointegration vectors, Journal of Economic Dynamics and Control, 12, 231-254.

Lütkepohl, H. (2005). New Introduction to Multiple Time Series Analysis, Springer-Verlag, Berlin.

Seo, B. (2007). Asymptotic distribution of the cointegrating vector estimator in error correction models with conditional heteroskedasticity, Journal of Econometrics, 137, 68-111.

Seong, B. (2013). Semiparametric selection of seasonal cointegrating ranks using information criteria, Economics Letters, 120, 592-595.

Seong, B., Cho, S. and Ahn, S.K. (2006). Maximum eigenvalue test for seasonal cointegrating ranks, Oxford Bulletin of Economics and Statistics, 68, 497-514.

van der Weide, R. (2002). GO-GARCH: A multivariate generalized orthogonal GARCH model, Journal of Applied Econometrics, 17, 549-564. 